ESAIM: PROCEEDINGS AND SURVEYS, September 2014, Vol. 45, p. 369-378

J.-S. Dhersin, Editor

\title{
TOPOLOGY OPTIMIZATION OF CONVECTIVE LAMINAR HEAT TRANSFER
}

\author{
Gilles Marck $^{1}$, Maroun Nemer $^{1}$ and Jean-Luc Harion ${ }^{2}$
}

\begin{abstract}
The topology optimization of systems subject to a fluid flow shows a wide potential for designing optimal and innovative structures. The present works apply the concepts of shape optimization and shape derivative to laminar flows (Navier-Stokes) coupled with heat transfers. In addition to the direct model introduction, a special attention is given to the bi-objective optimization problem and to the algorithm carried out to solve it. The shape derivative is computed thanks to an adjoint state based on a discretization process using the finite volume method. The results show the optimal path of a fluid within a solid domain, taking into account both objectives relative to the minimization of the viscous dissipation and to the maximization of the thermal heat transfer.

Résumé. L'optimisation topologique de systèmes sujets à un écoulement fluide présente un potentiel important pour la conception de structures optimales et innovantes. Les travaux exposés appliquent les concepts de l'optimisation de forme et de la dérivée de forme à l'optimisation d'écoulements laminaires (Navier-Stokes) couplés au transport de la chaleur. Outre la présentation du modèle direct, une attention particulière est donnée au problème d'optimisation qui est par nature bi-objectif et à l'algorithme mis en œuvre pour le résoudre. La dérivée de forme est établie au moyen d'un état adjoint basé sur une discrétisation en volumes finis. Les résultats présentés illustrent l'optimisation d'un parcours fluide au sein d'un domaine solide, en prenant en compte les objectifs liés à la minimisation de la dissipation visqueuse et à la maximisation du transfert thermique.
\end{abstract}

\section{INTRODUCTION}

Among the numerical methods used to solve engineering issues, multidisciplinary shape optimization became a wide-ranging tool for addressing realistic problems. In particular, the so-called topology optimization method gains in popularity during the last decade, first for tackling structural optimization problem, then for optimizing devices subject to electromagnetism fields or fluid flows. This class of methods, which is gradient-based, directly looks for the best layout of material, independently of a pre-established shape or configuration. Therefore, the optimal structure arises from the optimization process, without any mindset regarding the final configuration, unlike the parametric optimization methods for instance.

The shape optimization problems in fluid mechanics are very important and gave rise to many works. Most often, these works have a numerical character due to the intrinsic difficulty of the Navier-Stokes equations. For a first bibliography on the topic, we refer to [1-4]. In order to be able to define shape derivatives and study the sensitivity of shape functionals, we need to construct such a structure for the shape spaces. In the literature, this is done by considering perturbations of an initial domain; see for example [5,6].

The strategy pursued in this proceeding consists of building a size optimization problem from the topology one $[7,8]$, taking advantage of the discretization process used to solve the partial differential equations governing

${ }^{1}$ Mines ParisTech, CES, 60 Bd St Michel 75272 Paris Cedex 06, France. e-mail: gilles.marck@ljll.math.upmc.fr

2 Univ Lille Nord de France, F-59000 Lille, Mines Douai, EI, F-59500 Douai, France

(C) EDP Sciences, SMAI 2014 
the physical problem under consideration. The numerical background to perform such optimization has been developed for Stokes flows [1,9]. These works have been followed by several other studies applying the same approach to different flow regimes such as Darcy-Stokes $[10,11]$ or Navier-Stokes $[12,13]$. These numerical investigations mainly aim at minimizing the mechanic power dissipated by the fluid motion, in other words minimizing the pressure drop between the inlet and outlet flows, by reaching an optimal configuration of solid and fluid regions. Theses results agree with several theoretical studies about the optimality conditions and the existence of such shape optimization problems (see for instance [14-16]).

Topology optimization has also been performed to reach optimal designs in the frame of conductive heat transfers. Evgrafov et al. analyzed the convergence of such problems [17] and numerical solutions have been established for multi-objective optimization, aiming for instance at minimizing both mean and variance temperature of conductive fields [18]. These results underline the possibility to reach a set of Pareto optimal solutions from the topology optimization approach.

Studying separately flow configuration and conductive heat transfer problems naturally leads to the next optimization challenge: bringing together both problems to optimize at the same time heat and mass transfer objectives. Dede studied the optimization of a bi-dimensional geometry involving both Navier-Stokes and transport equations, solved with COMSOL commercial software [19]. This first results have been followed by Kontoleontos and Papoutsis-Kiachagias' works (et al.), who extended the numerical model to turbulent flows thanks to the Spalart-Allmaras assumption [20,21]. However, they do not consider in their works the temperature field within the solid domain, which is numerically set to a constant value.

This proceeding pursues the same strategy as Dede's one, namely optimizing both fluid and solid domains by adjusting their pseudo-porosity and thermal conductivity. Therefore, two aspects of the heat flux transport are optimized: pure conduction in the solid domain, and conducto-convection in the fluid domain. After introducing the direct problem, the optimization problem and the main algorithm, details are given to deal with the multiobjective nature of the heat and mass transfer problem, in a similar manner to [18]. Then, a study case tackles a bi-objective optimization problem, looking for the Pareto set of solutions minimizing the viscous dissipation while maximizing the heat transfer.

\section{Heat AND MASS TRANSFER OptimizATiON}

\subsection{Direct problem}

Let $\Omega$ be a bounded connected domain of $\mathbb{R}^{2}$ and let us assume that its boundary denoted $\partial \Omega$ is at least $C^{2}$. Let us denote by $\mathbf{n}$ the outward unit normal vector. The domain $\Omega$ is made of a fluid and solid subdomains, respectively denoted $\Omega_{f}$ and $\Omega_{s}$, such as $\Omega_{f} \cap \Omega_{s}=\emptyset$. In the whole paper, the notation $\chi_{\Omega_{f}}$ stands for the characteristic function of a Lebesgue measurable set $\Omega_{f}$. Figure 1 shows such a configuration, with a random distribution of solid and fluid subdomains. Fluid is assumed to be Newtonian and incompressible, under steady state laminar regime. It is characterized by its dynamic viscosity $\mu$, its thermal heat capacity $C_{p}$, its density $\rho$ and its thermal conductivity $k_{f}$. Conversely, solid subdomains are characterized with a thermal conductivity $k_{s}$. In addition, $\partial \Omega$ is subject to different boundary conditions: inlet flows with parabolic profile $\mathbf{u}_{i}$ at constant temperature $T_{i}$, outlet flows with parabolic profile $\mathbf{u}_{o}$ with zero temperature gradient $(\partial T / \partial n=0)$, and walls with a no-slip assumption $\left(\mathbf{u}_{w}=0\right)$ at constant temperature $T_{w}$ or with an adiabatic behaviour $(\partial T / \partial n=0)$.

The optimization problem aims at optimizing one or several objective functions by shrewdly allocating both $\Omega_{f}$ and $\Omega_{s}$ subdomains. The approach chosen here locally adds an internal friction force between the fluid and a small obstacle in the same way as the Darcy's law within a porous media [9]. This new inverse permeability coefficient, denoted $\alpha$, ranges from 0 to a sufficiently large value to create a momentum sink modeling the behavior of solid subdomains. This additional term allows solving only one set of equations for both subdomains, if the $\alpha$ and $k$ coefficients of Navier-Stokes and energy transport equations are properly fitted. Following the assumptions made in this section, these equations write 


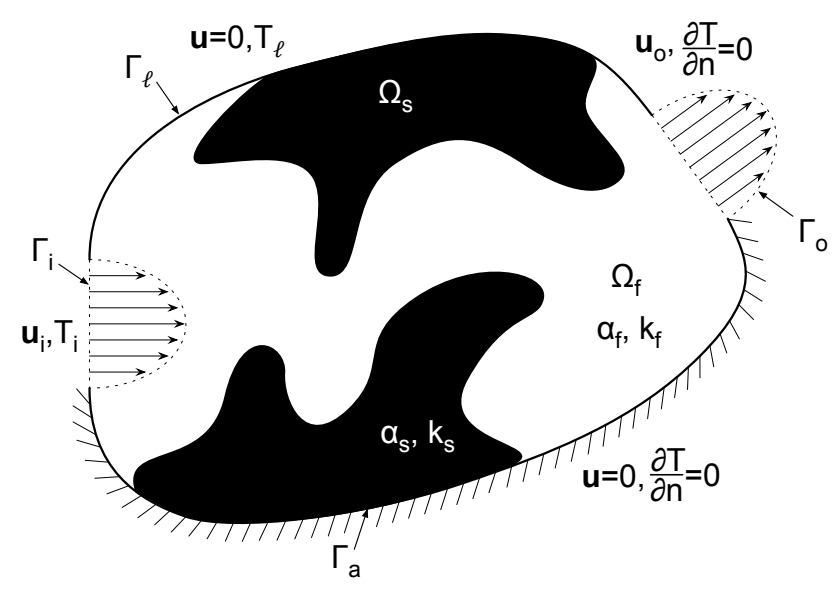

FiguRE 1. The fluid subdomain $\Omega_{f}$ (white area) is characterized by a laminar flow taking place between its inlet and outlet boundaries $\left(\Gamma_{i}\right.$ and $\Gamma_{o}$ ). The solid subdomain $\Omega_{s}$ (black areas) has a thermal conductivity $k_{s}\left(\neq k_{f}\right)$ and is bounded by either $\Gamma_{a}$ or $\Gamma_{\ell}$.

$$
\begin{array}{ll}
\nabla \cdot \mathbf{u}=0 & \text { in } \Omega \\
\rho(\mathbf{u} \cdot \nabla) \mathbf{u}+\alpha\left(\chi_{\Omega_{f}}\right) \mathbf{u}=-\nabla P+\mu \nabla^{2} \mathbf{u} & \text { in } \Omega \\
\rho C_{p}(\mathbf{u} \cdot \nabla) T=\nabla \cdot\left(k\left(\chi_{\Omega_{f}}\right) \nabla T\right) & \text { in } \Omega \\
\mathbf{u}=\mathbf{u}_{i}, \quad T=T_{i} & \text { on } \Gamma_{i} \\
\mathbf{u}=\mathbf{u}_{o}, \quad \nabla T \cdot \mathbf{n}=0 & \text { on } \Gamma_{o} \\
\mathbf{u}=\mathbf{0}, \quad T=T_{\ell} & \text { on } \Gamma_{\ell} \\
\mathbf{u}=\mathbf{0}, \quad \nabla T \cdot \mathbf{n}=0 & \text { on } \Gamma_{a}
\end{array}
$$

where $\alpha\left(\chi_{\Omega_{f}}\right)$ and $k\left(\chi_{\Omega_{f}}\right)$ are domain dependent. If $\mathbf{x} \in \Omega_{f}$, then $\alpha(1)=0$ and $k(1)=k_{f}$. Conversely, if $\mathbf{x} \in \Omega_{s}$, then $\alpha(0) \rightarrow \infty$ and $k(0)=k_{s}$. Further details about the definition of $\alpha$ and $k$ are providing in the next section. This set of equations is discretized with the finite volume method and the so-called SIMPLER algorithm is used to solve the pressure-velocity coupling [22]. Furthermore, an additional correction is included to properly take into account the viscous dissipation caused by the wall shear stress along the solid domain (see [23] for details).

\subsection{Optimization problem}

The heat and mass transfer problem introduced as so far is completed with objective functions depending explicitly on $\mathbf{u}, P$ and $T$, and implicitly on $\chi_{\Omega_{f}}$. The first objective function $J_{d}$ is relative to minimizing the mechanic power dissipated by the fluid through the domain $\Omega$ and can be computed thanks to the total pressure losses

$$
J_{d}(\mathbf{u}, P)=\int_{\Gamma}-\left(P+\frac{1}{2} \rho|\mathbf{u}|^{2}\right) \mathbf{n} \cdot \mathbf{u} d \Gamma
$$

if the boundary $\Gamma$ is smooth enough, since the trace of pressure $P$ must be defined on it. The second objective function $J_{e}$ aims at maximizing the thermal power recovered from the domain $\Omega$, by means of the inlet and outlet flow boundary conditions. This net thermal power is given by

$$
J_{e}(\mathbf{u}, T)=\int_{\Gamma}\left(\rho C_{p} T\right) \mathbf{n} \cdot \mathbf{u} d \Gamma .
$$


The challenge is to conjointly optimize $J_{d}$ and $J_{e}$ objective functions in order to reach a configuration being a trade-off between both objectives. This means that further maximization of the thermal power extracted by the fluid involves increasing the mechanical losses, and conversely. This strategy leads to multiple solutions, referred to as the Pareto front. Therefore, the optimization problem is bi-objective and can be stated as

$$
\inf _{\chi_{\Omega_{f}} \in \mathcal{A}_{\bar{\varphi}}} J_{d}(\mathbf{u}, P), \sup _{\chi \Omega_{f} \in \mathcal{A}_{\bar{\varphi}}} J_{e}(\mathbf{u}, T)
$$

subject to Equation (1) and where $\mathcal{A}_{\bar{\varphi}}=\left\{\chi_{\Omega_{f}} \in L^{\infty}(\Omega,\{0,1\}),\left|\Omega_{f}\right| \leq \bar{\varphi}|\Omega|\right\}$ with $0 \leq \bar{\varphi} \leq 1$. $\bar{\varphi}$ is a volumic constraint ensuring that $\Omega$ is not only made of fluid, which is the best trivial solution for most of problems tackling the minimization of the viscous dissipation.

A suitable strategy allowing reaching the Pareto front lies on a linear combination of both objectives, which is also known as the aggregated objective function method. This approach can only generates the convex part of the Pareto frontier: this feature is assumed during the computational phase and will be checked afterwards. However, before linearly combining the objective functions, they must be rescaled to reach an homogenous distribution of samples along the Pareto frontier. This rescaling writes

$$
\hat{J}=\frac{J-\underline{J}}{\bar{J}-\underline{J}}
$$

where $\underline{J}$ and $\bar{J}$ respectively stands for the inferior and superior bounds of $J$ functions. Consequently, before computing an optimal design for an aggregated objective function made of $n$ objectives, it is required to solve $2 n$ optimization problems. Then, the Pareto front may be outlined by minimizing $J$ with several different values of $w \in[0,1]$, where $J$ writes

$$
J(\mathbf{u}, P, T)=-w \hat{J}_{e}(\mathbf{u}, T)+(1-w) \hat{J}_{d}(\mathbf{u}, P) .
$$

Note that the thermal objective $\hat{J}_{e}$ is negatively weighted because it must be maximized.

It is well known that the solutions of infinite dimensional parametric problem such as the one introduced in Equation (1) do not necessarily exist (see $[3,8]$ ). To overcome this difficulty, a relaxation procedure is carried out: it mainly aims at extending the admissible set of solutions by replacing $\chi_{\Omega_{f}} \in L^{\infty}(\Omega,\{0,1\})$ by $\eta \in L^{\infty}(\Omega,[0,1])$. Consequently, $\alpha$ and $k$ continuously depend on the function $\eta$ and this feature can be exploited to cleverly control the convergence process. A penalization parameter $p$ is introduced to form the following penalization function [9]

$$
\sigma(\eta, p)=\sigma_{s}+\left(\sigma_{f}-\sigma_{s}\right) \eta \frac{1+p}{\eta+p} \quad \text { with } \quad 0 \leq \eta \leq 1 \quad \text { and } \quad p>0
$$

where $\sigma$ is a generic functional standing for either $\alpha$ or $k$. The penalization parameter $p$ masters the convexity of this function, as shown in Figure 2 and allows tuning the convergence of the optimization problem. For instance, during the first iterations of the algorithmic scheme, $p$ starts with a value close to 0.01 , and increases to 1 when the solution is almost converged. Combined with other numerical tools, speeding up or slowing down the convergence by means of $p$ allows escaping from local optima. Note that from this point, since the design parameters are continuous, domain includes both solid and fluid elements, but also composite materials acting like an artificial porous media.

Behind the non-existence issue, the second advantage of the relaxation step is to allow computing the shape derivative from the parametric formulation. Let denote $\langle d J(\eta), h\rangle$, the differential of $J$ with respect to $\eta$ in direction $h$. This derivative is determined thanks to an adjoint state built from the discrete form of Problem (1): the corresponding numerical strategy is detailed in [23] and is beyond the scope of this proceeding. However, before being used in the optimization process, the shape derivative is filtered with a convolution product 


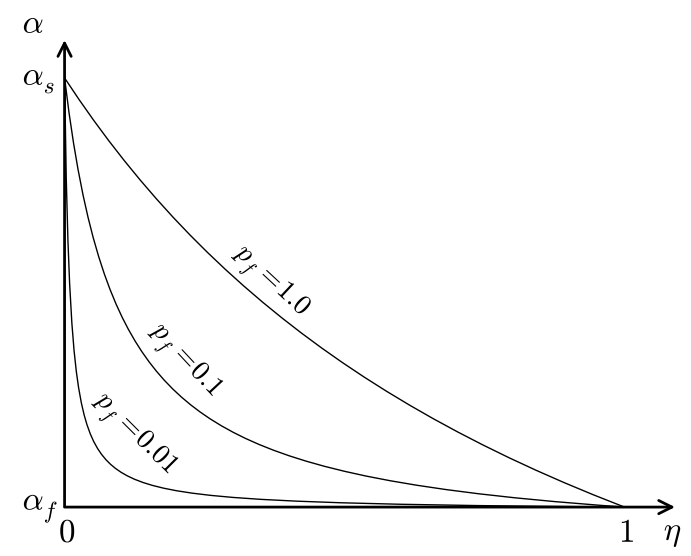

(A) Fluid convergence $\alpha$.

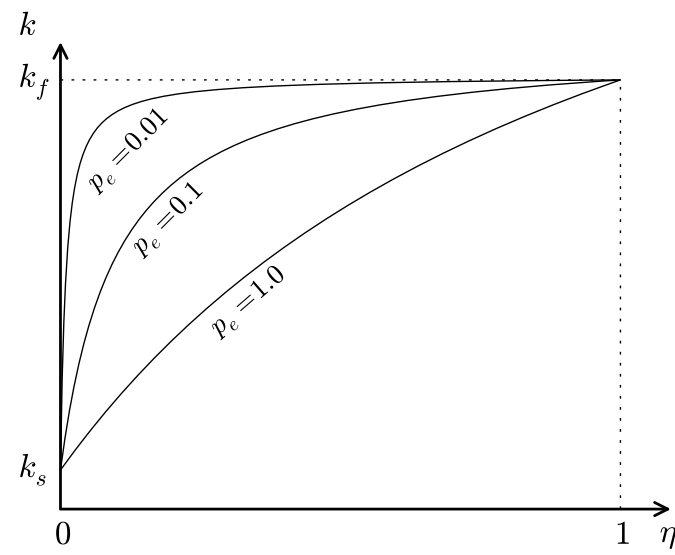

(B) Thermal convergence $k$.

Figure 2. Convergence control strategy.

involving the discrete field $\eta$ as follows (see [7]),

$$
\left\langle\widetilde{d J}\left(\eta_{\ell}\right), h\right\rangle=\frac{\sum_{\ell=1}^{N} \eta_{\ell} H_{\ell}\left\langle d J\left(\eta_{\ell}\right), h\right\rangle}{\eta_{\imath} \sum_{\ell=1}^{N} H_{\ell}}
$$

where $N$ is the number of discrete elements satisfying $\left|\mathbf{x}_{\imath}-\mathbf{x}_{\ell}\right| \leq r$ and $H_{\ell}=r-\left|\mathbf{x}_{\imath}-\mathbf{x}_{\ell}\right|$. This step aims at avoiding the so-called checkerboard problems leading to unrealistic optimal configurations due to the discretization process.

\subsection{Algorithm}

The main sequence optimizing the aggregated objective function $J$ is detailed in Algorithm 1. Its structure has been adapted from the SIMP method (see [7]), but with several adjustments to take into account the convergence issues coming from the fluid nature of the problem. The computation of the objective function is not detailed here: the instruction $J \leftarrow J\left(\eta, p_{f}, p_{e}\right)$ means that the finite volume solver computes the $\mathbf{u}, P, T$ solution, as well as the objective functions. The algorithm itself is based on an inner loop, which is nested within an outer loop. The inner loop aims at solving the optimization problem for a given state of the penalization parameters, whereas the outer loop controls them and is responsible for the convergence test leading to exit the whole algorithm.

The inner loop sequentially computes the shape derivative $\langle d J(\eta), h\rangle$, its filtered form $\left\langle\widetilde{d J}\left(\eta_{\imath}\right), h\right\rangle$, as well as the problem constraints and their gradient, respectively denoted $\varphi(\eta)$ and $\langle d \varphi(\eta), h\rangle$. These variables becomes the inputs of the Method of Moving Asymptotes (MMA), which belongs to a comprehensive class of gradient optimization methods based on conservative convex approximations. MMA has been shown to be particularly efficient for solving inequality-constrained nonlinear programming problems, especially in the frame of structural and parametric optimization $[24,25]$. It has several internal mechanisms which allow avoiding the local optima for some types of convergence patterns. Once a new $\eta$ state have been generated, the Navier-Stokes and transport equations are solved again and the algorithm loops until the convergence criterion $\epsilon_{i}$ is satisfied.

The outer loop is indexed by means of an integer $j$ ranging from 0 to a fixed number of iterations. This index drives four inner different parameters: the residual $\epsilon_{i}$, the penalization parameters $p_{f}$ and $p_{e}$, and the radius 
$r$ of the numerical filter applied to the shape derivative. If the convergence path defined by these parameters is carefully chosen, the convergence process can be drastically speeded up. For instance, during the first outer iterations, the inner loop does not need to fully converge, since the penalization parameters are close to 0 and force the solution towards porous areas. These first steps can be seen as an additional initialization procedure. Furthermore, two penalization parameters are defined for the fluid and for the energy equations, respectively denoted $p_{f}$ and $p_{e}$, in order to control separately the convergence of each phenomenon.

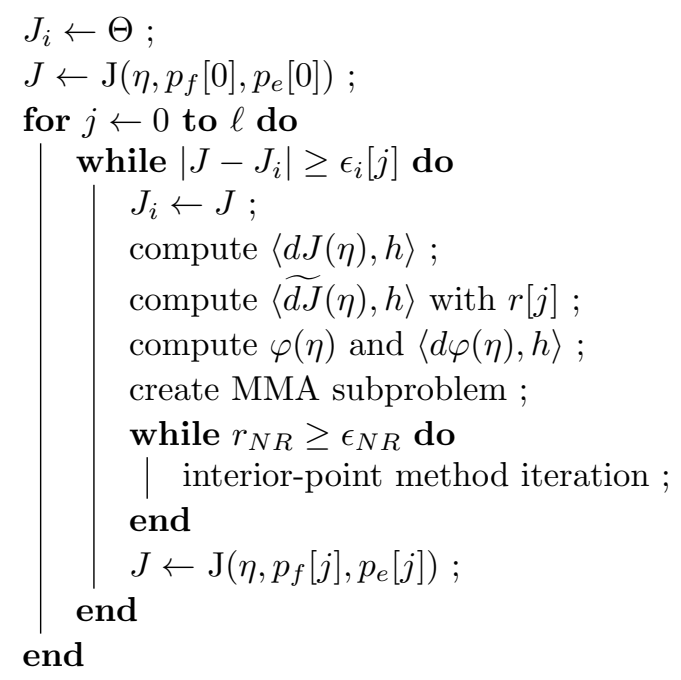

Algorithm 1: Shape optimization algorithm.

\section{Results}

First, the numerical result analysis investigates the suitability of the finite volume method to deal with the additional pseudo-porosity term introduced in Equation (1), without tackling the heat transfer problem. Then, the problem is solved from a multi-objective point of view in order to compute the Pareto front. The case studied is a square-shaped domain of side $\ell=0.1 \mathrm{~m}$, displayed in Figure 3. The inlet and outlet flows are horizontally lined up in front of each other, with the same parabolic $\mathbf{u}$-velocity profiles. The inlet flow temperature is set to $T_{i}=0{ }^{\circ} \mathrm{C}$ and the vertical walls are assumed to be adiabatic. The horizontal walls closing the domain are subject to a constant temperature $T_{w}=10^{\circ} \mathrm{C}$. The Reynolds number of this system, computed on the basis of the characteristic dimension $\ell / 5$ and the average inlet velocity, is $R e=3$. The thermal parameters are the following: $k_{s}=10 \mathrm{~W} /(\mathrm{m} . \mathrm{K}), k_{f}=1.0 \mathrm{~W} /(\mathrm{m} . \mathrm{K})$, and $C_{p}=5.0 \mathrm{~kJ} /(\mathrm{kg} . \mathrm{K})$. The domain is discretized with $100 \times 100$ design elements and the volume constraint insures that $\left|\Omega_{f}\right|<0.4|\Omega|$.

\subsection{Minimization of viscous dissipation}

Figure 4 displays the results for $w=0$, minimizing the power lost by viscous dissipation within the fluid domain. As underlined by Figure 4a, the flow straightforwardly joins the inlet and outlet boundary conditions, with the largest fluid domain as possible. This aims at letting $\Omega_{f}$ being as wide as allowed by the constraint $\varphi(\eta) \leq \bar{\varphi}$ in order to minimize the shears inside the fluid flow. Figure $4 \mathrm{~b}$ gives details about the pressure field within the $\Omega_{f}$ domain, which mainly follows an horizontal gradient, in a similar way as the Poiseuille flow for instance. Figure 4c highlights both physical configurations for the heat transfer:

- conduction within the solid domain, which mainly remains at temperatures close to $T_{w}$;

- conducto-convection within the fluid flow, which mainly transports the energy in its lower and upper boundary layers. These are distant from the Dirichlet boundary conditions having a high thermal potential with regards to the objective function $J_{e}$. 


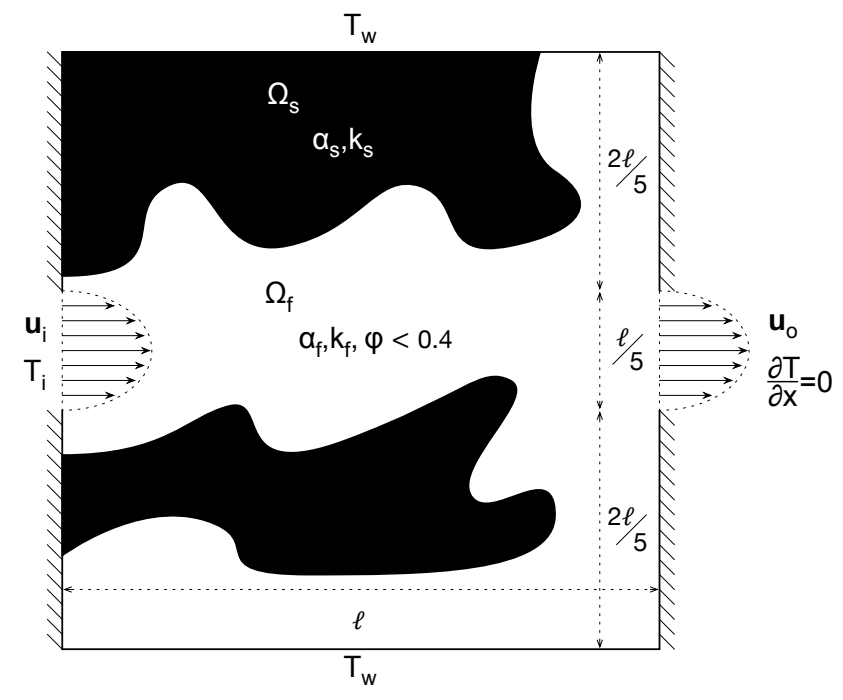

FiguRE 3. Single pipe configuration.

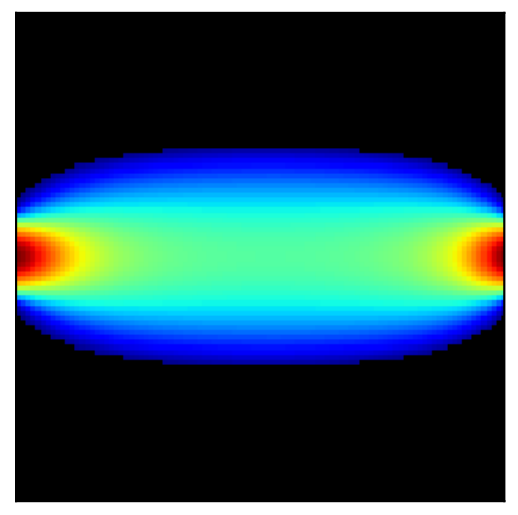

(A) $0 \leq|\mathbf{u}| \leq 1 \times 10^{-3} \mathrm{~m} / \mathrm{s}$

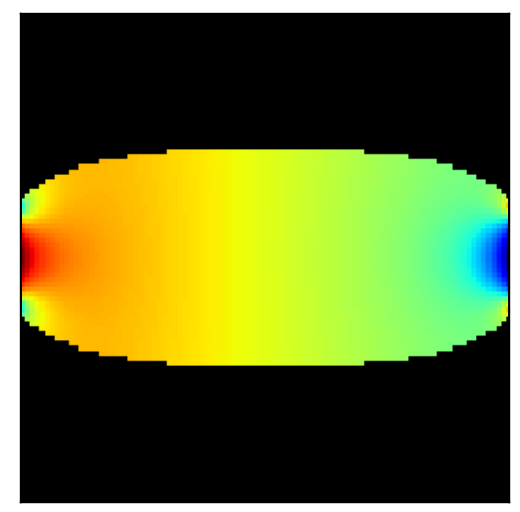

(в) $-2.1 \leq P \leq 0 \times 10^{-3} \mathrm{~Pa}$

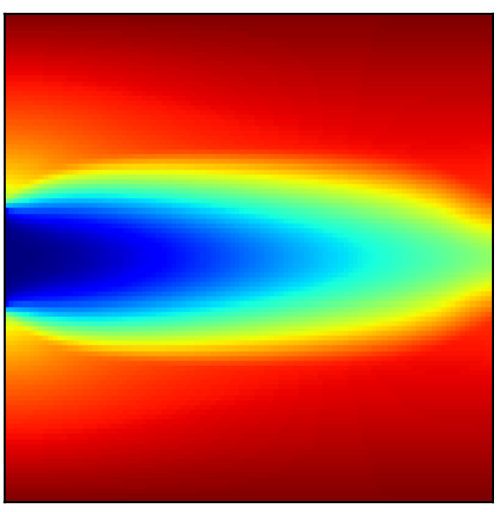

(C) $0 \leq T \leq 10^{\circ} \mathrm{C}$

Figure 4. Minimization of the viscous dissipation in $\Omega$.

\subsection{Pareto front}

The observation of the thermal pattern in Fig. 4c suggests that shape optimization is a relevant approach for maximizing $J_{d}$. Figure 5 displays the bi-objective optimization results that are reached by gradually increasing the weighting $w$ and after the rescaling process detailed in Equation (5). Four main classes of solution can be distinguished from their topology:

- For $w=0$, the fluid is transported through a straight pipe, as detailed in section 2.1.

- For $0.06 \leq w \leq 0.12$, a solid core takes place at the center of the domain, splitting the fluid subdomain into a lower and an upper flows, as shown from Figures 5b to 5d. This moves both flows towards the lower and upper walls at constant temperature $T_{w}$, heating up the fluid without the temperature losses induced by the heat flux conduction through the solid domain. The more the $w$ weighting increases, the more the central core width increases. 


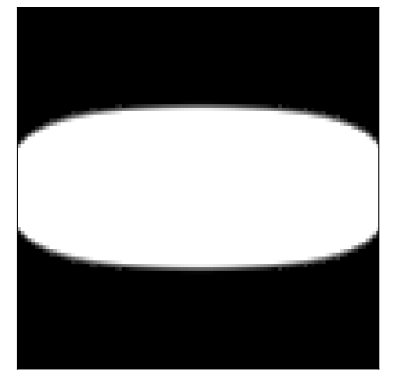

(A) $w=0.0$

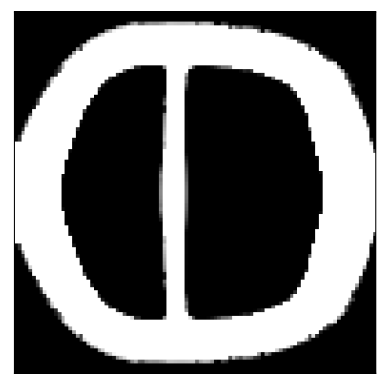

(E) $w=0.26$

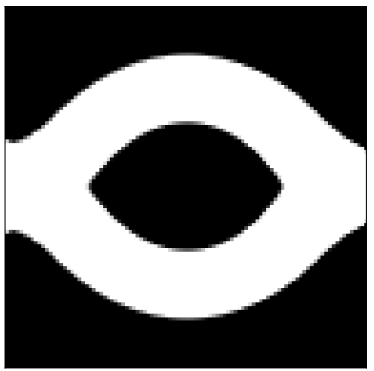

(в) $w=0.03$

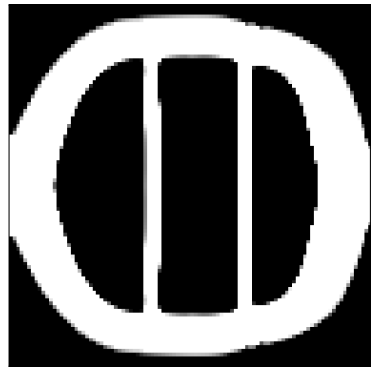

(F) $w=0.28$

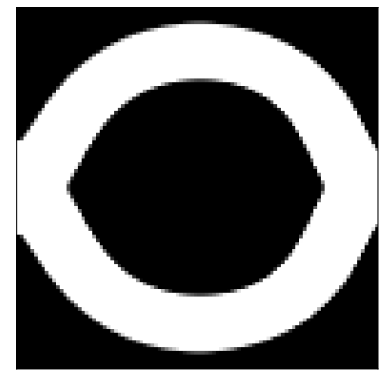

(C) $w=0.06$

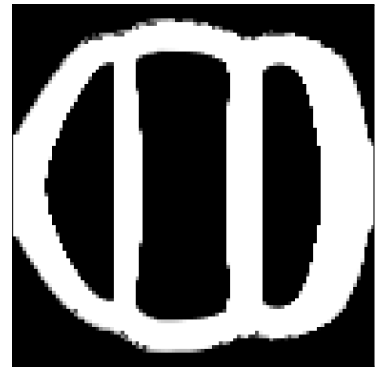

(G) $w=0.60$

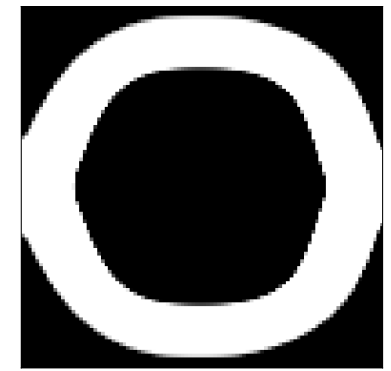

(D) $w=0.12$

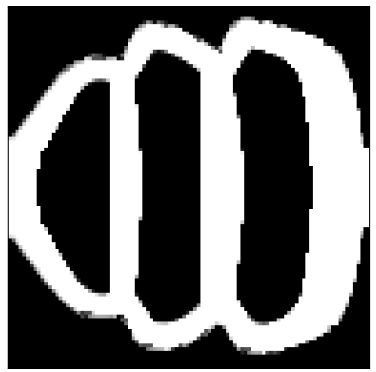

(н) $w=0.75$

FIGURE 5. Optimal structures depending on $w$ weighting.

- For $0.26 \leq w \leq 0.60$, the central core is vertically split into several sub-cores, as shown from Figures 5e to $5 \mathrm{~g}$. This behavior breaks the horizontal temperature gradient through the solid core, by inserting one or more strips of fluid acting like a heat insulation material. Further details are provided about this phenomenon in section 2.3 .

- For $w=0.75$, the surface of solid and fluid interfaces closed to the boundary conditions $T_{w}$ are artificially increased by small solid inserts. This feature increases the main surfaces taking part into the heat transfer process.

Figure 6 shows the Pareto front of the current example, which is convex and can be reached from an aggregated objective function. From a physical point of view, the main point highlighted by this set is the optimality of a few solutions: the weightings around $w=0.5$ provide structures having both $J_{d}$ minimum and $J_{e}$ maximum close to their single objective optimization. In other words, these configurations are acceptable trade-offs between the flow derivation, which increases the viscous dissipation, and the core fragmentation, which increases the thermal heat transfers.

\subsection{Physical analysis}

Figure 7 demonstrates the interest of splitting the central core in several different parts. Since $k_{f}<k_{s}$, the fluid areas having a zero-velocity field can be seen as an insulation material from the heat flux point of view. From a conductive point of view, the fluid strips act as large thermal resistances for the heat flowing through the solid core. The central field temperature is deeply affected by these discontinuities, as underlined by Figure $7 \mathrm{~b}$. Without the strips of fluid, an horizontal temperature gradient would take place through the core, driving the heat flux from the near outlet flow area to the near inlet one. Consequently, the outlet flow would be cooled by conduction through the central core, penalizing the objective function $J_{e}$. In other words, dividing the central part allows the temperature homogenization for each of its subset by restricting heat conduction, and ensures that both extreme parts are mainly under the influence of their closest thermal boundary condition. 


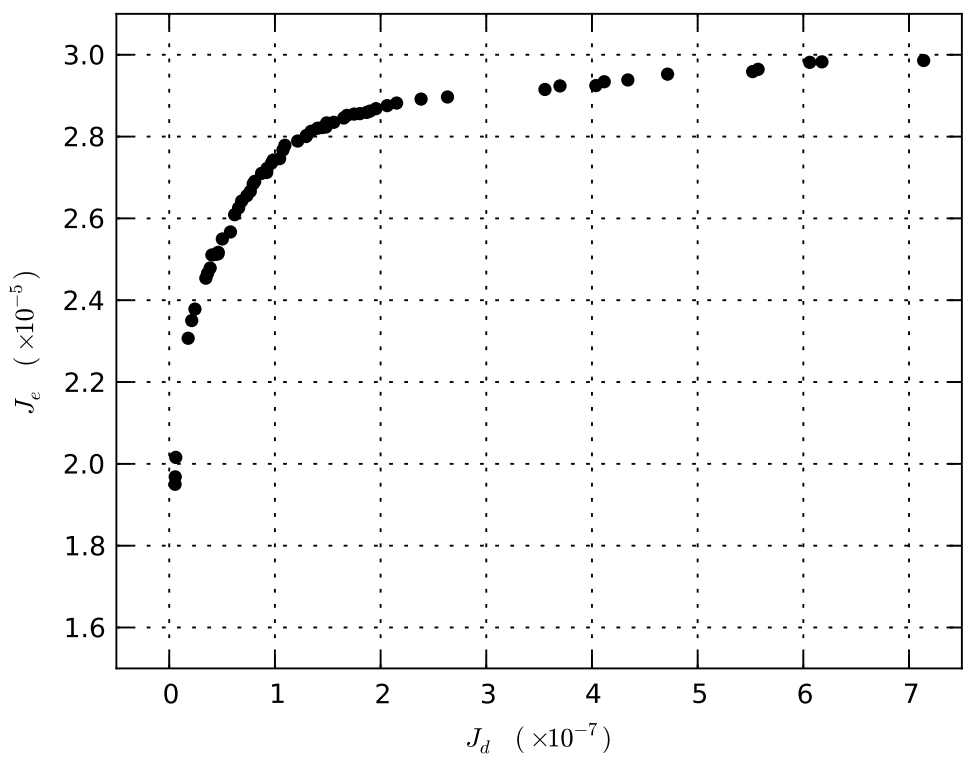

Figure 6. Pareto front.

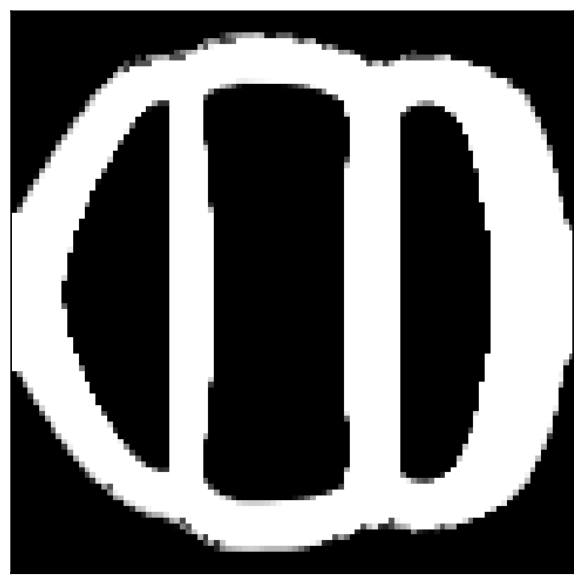

(A) $\eta$

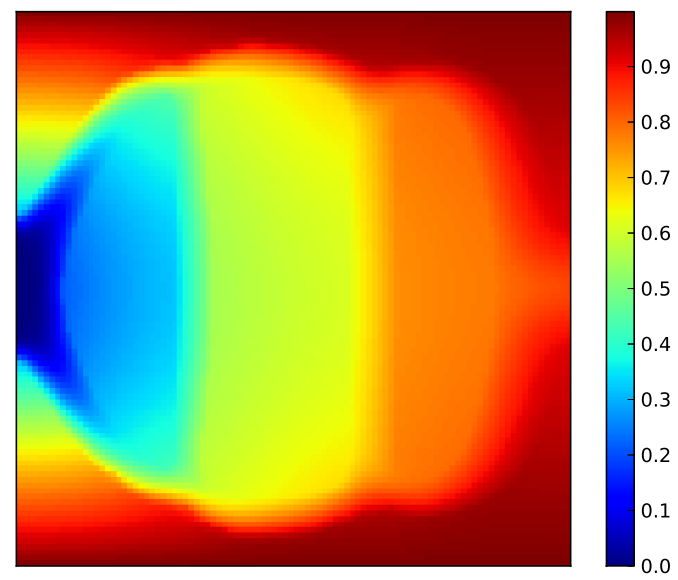

(в) $\left(T-T_{i}\right) /\left(T_{w}-T_{i}\right)$

Figure 7. Analysis of the core fragmentation from a thermal point of view.

\section{CONCLUSION}

In this proceeding, we show that a trade-off between fluid and thermal objective functions could be reached on the basis of numerical investigations and that these solutions are not trivial from a structural point of view. The investigated example illustrates the main problematic of heat and mass transfers, consisting in increasing the thermal heat exchange with the fluid, while reducing as far as possible the power required to set it in motion. In this case, the aggregated objective function approach is proven to be a suitable strategy since the Pareto front of the problem is almost fully convex. 
One interesting point underlined by Figure 7 is the presence of homogenized areas along the fluid/solid interfaces. Indeed, even if this solution seems to be fully converged, the solid boundaries remain rough: they are made of an artificial porous media. The split core may also be seen as a numerical manifestation of the homogenization process, since a core made of an infinite succession of vertical solid and fluid layers would perform better than any other one, for the same reasons as those explained in section 2.3. The numerical observation of this homogenization is made possible by the problem relaxation. This should be investigated from a theoretical point of view, especially because the numerical experiments tend to show that the more the weighting of the thermal objective is larger, the more the homogenization process become prominent.

\section{REFERENCES}

[1] B. Mohammadi and O. Pironneau. Applied Shape Optimization for Fluids. Numerical Mathematics and Scientific Computation. Oxford University Press, USA, 2001.

[2] G. Allaire. Shape Optimization by the Homogenization Method, volume 146 of Applied Mathematical Sciences. Springer, 2002.

[3] A. Henrot and M. Pierre. Variation et optimisation de formes: Une analyse géométrique. Springer, 2005.

[4] E. Feireisl. Shape Optimization in Viscous Compressible Fluids. Applied Mathematics and Optimization, 47(1):59-78, 2008.

[5] F. Murat and J. Simon. Sur le contrôle par un domaine géometrique. Publication du Laboratoire d'Analyse Numérique de l'Université Paris 6, 189, 1976.

[6] J. Sokolowski and J.P. Zolésio. Introduction to Shape Optimization: Shape Sensitivity Analysis, volume 16. Springer-Verlag, 1992.

[7] M. Bendsoe and O. Sigmund. Topology Optimization: Theory, Methods and Applications. Springer, 2 nd edition, 2003.

[8] G. Allaire. Conception optimale de structures. Mathématiques et Applications. Springer, 2006.

[9] T. Borrvall and J. Petersson. Topology optimization of fluids in Stokes flow. International Journal for Numerical Methods in Fluids, 41(1):77-107, 2003.

[10] J. Guest and J. Prévost. Topology optimization of creeping fluid flows using a Darcy-Stokes finite element. International Journal for Numerical Methods in Engineering, 66:461-484, 2006.

[11] N. Wiker, A. Klarbring, and T. Borrvall. Topology optimization of regions of Darcy and Stokes flow. International Journal for Numerical Methods in Engineering, 69(7):1374-1404, 2007.

[12] A. Gersborg-Hansen, O. Sigmund, and R. Haber. Topology optimization of channel flow problems. Structural and Multidisciplinary Optimization, 30(3):181-192, 2005.

[13] C. Othmer. A continuous adjoint formulation for the computation of topological and surface sensitivities of ducted flows. International Journal for Numerical Methods in Fluids, 58(8):861-877, 2008.

[14] A. Henrot and Y. Privat. What is the optimal shape of a pipe? Archive for Rational Mechanics and Analysis, 196(1):281-302, 2010.

[15] X. Dubois De La Sablonière, B. Mauroy, and Y. Privat. Shape minimization of the dissipated energy in dyadic trees. Discrete and Continuous Dynamical Systems - Series B, 16(3):767-799, 2011.

[16] M. Bergounioux and Y. Privat. Shape optimization with Stokes constraints over the set of axisymmetric domains. SIAM Journal on Control and Optimization, 51(1):599-628, 2013.

[17] A. Evgrafov, M. M. Gregersen, and M. P. Sorensen. Convergence of cell based finite volume discretizations for problems of control in the conduction coefficients. ESAIM: Mathematical Modelling and Numerical Analysis, 45(6):1059-1080, June 2011.

[18] G. Marck, M. Nemer, J.-L. Harion, S. Russeil, and D. Bougeard. Topology optimization using the SIMP method for multiobjective conductive problems. Numerical Heat Transfer, Part B: Fundamentals, 61(6):439-470, June 2012.

[19] E. Dede. Multiphysics topology optimization of heat transfer and fluid flow systems. In COMSOL Conference, 2009.

[20] E. M. Papoutsis-Kiachagias, E. A. Kontoleontos, A. S. Zymaris, D. I. Papadimitriou, and K. C. Giannakoglou. Constrained topology optimization for laminar and turbulent flows, including heat transfer. In CIRA, editor, EUROGEN, Evolutionary and Deterministic Methods for Design, Optimization and Control, Capua, Italy, 2011.

[21] E. A. Kontoleontos, E. M. Papoutsis-Kiachagias, A. S. Zymaris, D. I. Papadimitriou, and K. C. Giannakoglou. Adjoint-based constrained topology optimization for viscous flows, including heat transfer. Engineering Optimization, pages 1-21, 2012.

[22] H. Versteeg and W. Malalasekera. An introduction to computational fluid dynamics - The finite volume method. Pearson Education Limited, Harlow, 2nd edition, 2007.

[23] G. Marck, M. Nemer, and J.-L. Harion. Topology Optimization of Heat and Mass Transfer Problems: Laminar Flow. Numerical Heat Transfer, Part B: Fundamentals, 63(6):508-539, June 2013.

[24] K. Svanberg. The Method of Moving Asymptotes - A new method for structural optimization. International Journal for Numerical Methods in Engineering, 24:359-373, 1987.

[25] K. Svanberg. A class of globally convergent optimization methods based on conservative convex separable approximations. SIAM Journal on Optimization, 12(2):555-573, 2002. 